\title{
Light-based Internet of Things: Implementation of an Optically Connected Energy-autonomous Node
}

\author{
Amila Perera \\ Centre for Wireless Communications \\ University of Oulu \\ Oulu, Finland \\ amila.perera@oulu.fi \\ Juha Häkkinen \\ Centre for Wireless Communications \\ University of Oulu \\ Oulu, Finland \\ juha.hakkinen@oulu.fi
}

\author{
Marcos Katz \\ Centre for Wireless Communications \\ University of Oulu \\ Oulu, Finland \\ marcos.katz@oulu.fi \\ Esko Strömmer \\ VTT Technical Research \\ Centre of Finland \\ Espoo, Finland \\ esko.strommer@vtt.fi
}

\author{
Roshan Godaliyadda \\ Department of Electrical \\ and Electronic \\ Engineering \\ University of Peradeniya \\ Peradeniya, Sri Lanka \\ roshangodd@ee.pdn.ac.lk
}

\begin{abstract}
In the near future, with the development of 5G NR, a massive number of IoT nodes are expected to come online. Acquiring bandwidth from an overcrowded licensed radio frequency spectrum as well as overcoming battery life constraints will become the main challenges for these IoT based devices. As a promising solution for the above challenges, this work provides a feasibility study and an implementation framework for a visible light communication-based energy-autonomous IoT node. This work proposes the use of printed electronic technology to minimize the physical characteristics and cost of the IoT node. In the future, energy-autonomous IoT nodes could be available virtually on any surface. Whenever the node is exposed to light, it will be connected to internet.
\end{abstract}

Keywords-LIoT, VLC, IoT, Printed electronics, Indoor energy harvesting

\section{INTRODUCTION}

In the present world, as the demand for radio spectrum rapidly increases, facilitating sufficient bandwidth for these requirements becomes a challenge. This phenomenon is also defined as "spectrum crunch," which describes the unavailability of required spectrum bandwidth for the exponentially growing mobile users in the future [1]. Furthermore, with the evolution of $5 \mathrm{G}$ new radio, additional communication types such as machine-to-machine (M2M), device-to-device (D2D), device-to-everything (D2E), and internet of things (IoT) have emerged. Accommodating these massive communication bandwidths within the available radio frequency (RF) spectrum will be a truly challenging task. As a solution to this matter, switching from RF to optical communication is proposed here. Recent studies point out that the light fidelity (LI-FI) spectrum is a potential candidate spectrum for short-range communications. The LI-FI spectrum is made of a combination of both visible light and infrared (IR) spectrums. LiFi spectrum-based communication comes with many unique features such as virtually unlimited reuse, free license, inherent physical security, and robustness to electromagnetic interference [2]. In addition, visible light spectrum based visible light communication (VLC) technology has proven to reach several Gbps data rates in magnitude, which is well beyond the required data rate for most modern IoT applications [3]. With VLC-IoT optimized, efficient coding techniques such as Blinkcomm, the data rates can be further enhanced [4].

On the other hand, at the IoT node's design stage, the manufacturers need to overcome the unique design challenges of various IoT applications. Typically in IoT devices, key parameters such as energy consumption, battery capacity, cost per node, and data rates needed to be optimized according to the application requirement [5]. Usually, supercapacitors and batteries are commonly used to store energy for indoor and outdoor remote IoT applications. However, when their energy level degrades with time, battery or capacitor status monitoring and replacement are essential for these devices. These operations will be result in a costly and more time-consuming process, which depends on the accessibility of the device location [6]. The use of renewable energy can be effectively exploited to overcome power consumption and battery-related challenges. Studies suggest that renewable energy sources such as ambient light, vibration motion, thermal energy, and RF energy can be integrated with IoT nodes. Among these sources, ambient light has the ability to produce $5-30 \%$ efficiency, which is the highest compared to others [7]. According to [8], there are already RF communication, and traditional electronics-based energy harvesting solutions available for IoT nodes. When 
manufacturing the IoT nodes, the cost per node can be reduced at the production stage by introducing mass-production methods. Printed electronics (PE) is emerging as a manufacturing technology that can be also used for the largescale production of IoT device circuitries.

New developments in the literature combining wireless communications and PE are promising. The concept of Living Surfaces (LS) [9] is based on the combination of light-based IoT (LIoT) and other functionalities such as sensors, actuators, etc., all implemented on PE technology. The LIoT nodes are expected to communicate with VLC while being energyautonomous throughout their operation time. Based on LIoT concept, many possible applications can be developed. For instance, extremely low-cost active product labels could be designed to be connected to the network. Therefore, they could display information that can be remotely updated. This concept can also be effectively applied to a variety of portable devices, such as headphones and audio guides, to provide both wireless connectivity and energy harvesting based on light. The ability to support higher data rates in VLC means it can outperform the current widely using Bluetooth wireless technology data rates, leading to a high-quality user experience [10].

This paper describes a prototype implementation of the novel LIoT concept, where an energy-autonomous node is implemented. Critical components in the implementation use printed electronics technology. Initially, we study and identify the required available key technologies such as printed electronics and indoor energy harvesting technologies, which are essential for the LIoT implementation. Then, we design and implement the prototype system, including LIoT optimized VLC transmitter and energy-autonomous receiver nodes. Moreover, LIoT concept-friendly data transmitting schemes, system algorithms, and the viability of using general-purpose hardware and circuits for the implementation are discussed in the text. Finally, the performance of the transmission schemes, key subcircuits, and components (photodiode, energy harvesting unit, photovoltaic cell) of the prototype is evaluated under different possible scenarios, and results are discussed and verified. In addition, the feasibility of using high-efficiency perovskite and organic printed photovoltaic (PV) cell technologies for LIoT energy harvesting and their ability to receive signals were tested and verified based on the results.

The rest of the paper is organized as follows. In section II, we introduced the concept of LIoT and how it works. In section III, we describe the essential background information regarding the printed electronics technology. Section IV identifies critical requirements and challenges needed to be addressed when implementing the LIoT prototype. Section V describes the used algorithms, hardware circuit designs, and implementation process of the prototype. Finally, in Section VI and Section VII, we show, compare, and discuss the obtained results and verify the performance of the selected designs.

\section{Light-BASED IOT (LIOT)}

According to the concept of LIoT, the node is expected to communicate and energize by only using visible light. In this concept, light is exploited not only to illuminate the room but also to establish optical links to and from the LIoT node, as well as to power up this node by light energy harvesting. The transmitter is expected to transmit energy harvesting friendly modulated visible light waveform towards the LIoT node. The node is expected to receive the light signal through the optical channel, demodulate and decode the data while harvesting energy from the incident light energy on the surface. The general structure of LIoT node is illustrated as in Fig.1.

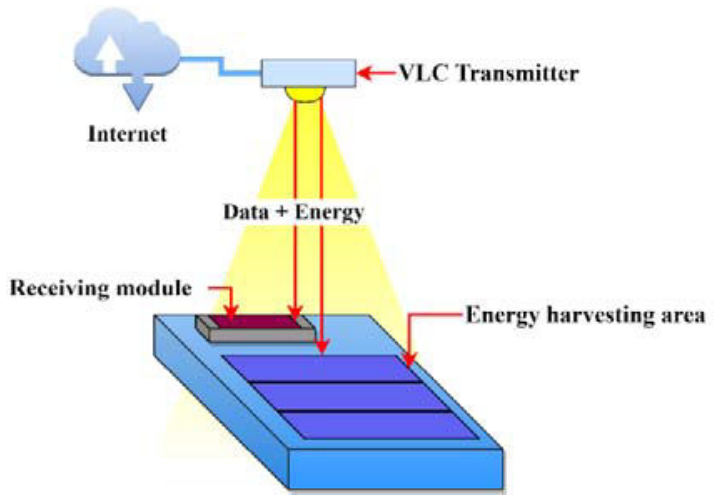

Fig.1. Structure of LIoT device

For the implementation of these types of nodes, it is important to use more compact sensors and circuitries in order to make it more feasible to integrate with today IoT applications. Technologies like printed electronics can be effectively used to fulfil this requirement.

\section{PRINTED ELECTRONICS}

Printed electronics (PE) is evolving as a promising technology to keep the applications of it are more compact, mechanically flexible and enable faster mass production capabilities at low cost. Compared to silicon chip fabrication, the non-recursive expenses can be reduced by using PE [11]. According to [12], there are two approaches to PE fabrication, namely, component type and system type. In component type, the designs are more focused only on fabricating flexible electronic components such as TFT transistors, resistors to perform similar to their analogue versions. The system type focuses on designing complete circuitries using PE technologies with unique PE characteristics. In addition, system type PE circuits can also be made using rigid electronic components, and these types of circuitries are known as flexible hybrid electronics (FHE).

When fabricating a PE application, electronic ink is deposited on the flexible substrate. The e-ink consists of functional materials, stabilizers, and rheological modifiers that give the expected characteristics for the printed element [13]. The substrate for the PE application is selected based on the expected mechanical requirements of the application. There are several methods to manufacture $\mathrm{PE}$ applications. In manufacturing, the roll-to-roll (R2R) printing concept is used to achieve a high production rate. In R2R, multiple sequences of dedicated printers used to print the electronics and store them back on a roller after production. These various methods can be classified into two main categories, such as contacting printing and non-contacting printing. Manufacturing methods such as screen printing, offset printing and flexography are an example 
of contacting printing. Printing methods such as Inkjet printing is a method where no contacts occur with the substrate when printing. Therefore, these methods are categorized as noncontacting printing methods.

The applications of PE are becoming very popular due to their unique characteristics over their traditional electronic counterparts. Among many PE applications, flexible PE displays are becoming a key PE application, which is an attractive way of enhancing user experience. Electrochromic displays made with PE technology are renowned due to their semi bi-stable low power consumption and fast production capabilities. On the other hand, PE-based 3rd generation PV cells are becoming popular due to their mechanical flexibility, transparent appearance, and equivalent performance to the traditional early generation rigid PV cells. According to [14], $\mathrm{R} 2 \mathrm{R}$, gravure printed perovskite cells have reached over $23 \%$ efficiency, which is a remarkable achievement for the PE-based PV technologies.

\section{REQUIREMENTS AND CHALLENGES}

The system's transmitters need to be designed to support required data rates while preserving the flicker-free indoor illumination. The parameters of the transmitter lighting source such as wavelength and intensity required to be tuned to provide sufficient light intensity for the power generation at the PV cells. The study carried out in [15] shows that wavelengths between 400-525nm can provide higher efficiency on PV cells than the rest of the spectrum. To find the Kelvin colour temperature corresponding to the wavelengths, Wien's Law can be used [16]. The authors describe that due to the electroluminescence process of LED illuminating, Wien's law might deviate from the ideal results, but it can be used for the emitting wavelength determination. The expression can be given as below

$$
\lambda_{\max }=\frac{2.898 * 10^{-3}}{T}
$$

In order to perform robustly under the typical indoor environment, a special noise elimination technique need to be introduced to the system. Several types of noise components can directly interfere with the VLC system. Thermal noise created by the random thermal motion of electrons in the load resistances and quantum shot noise, resulting from the dark current fluctuations in a photodiode-based VLC receiver, can be classified as noise originated from the electronic circuits. On the other hand, noise components from ambient sunlight and powerline frequency-based indoor luminaires can be classified as noise from the channel. In previous research works, it is proven that the approaches like frequency tuned Sallen-key high pass filter [17], adaptive minimum voltage cancellation [18], and Schmitt-trigger comparator arrangement [19] can be effectively used for noise mitigation.

In the receiver design, components like output unit and $\mathrm{MCU}$ need to be selected to minimize the energy consumption of the node. Bistable output technologies such as e-ink and electrochromic displays can be effectively used for low powered display purposes.

In the energy harvesting unit, the required amount of PV cell area can be reduced by keeping higher PV power conversion efficiency (PCE) for any given type of PV cell. By assuming the concept of ideal PV cell which made up of a constant current source, a diode, and a load, the parameters of PCE of an ideal PV can be expressed as below

$$
P C E=\frac{I_{S C} V_{O C} F F}{P_{\text {input }}},
$$

where

$$
\mathrm{FF}=\frac{I_{M P P} V_{M P P}}{I_{S C} V_{O C}}
$$

(MPP - Maximum power point, SC-short circuit, OC- Open circuit);

Furthermore, according to the general PV curve of a PV cell, it is important to maintain the terminal voltage closer to the opencircuit voltage $\left(\mathrm{V}_{\mathrm{OC}}\right)$ to get the maximum performance out from the PV cell. The I-V curve of the PV cell is illustrated in Fig.2.

On the other hand, it is vital to keep the current drawn by the load at a minimum to minimize the voltage drop at the terminal due to the series internal resistance of the PV cell. The selection of PV cell technology was made based on the efficiency under indoor illumination and mechanical characteristics. According to the study in [20], dye-sensitized PV shows $1.35 \%$ efficiency under indoor illumination, which outperforms the $0.45 \%$ efficiency of OPV. However, according to [14] specially treated perovskite cells are expected to achieve far better efficiency compared to others.

The capacitor type and values for the energy harvesting unit needs to be carefully selected. According to the [21], the charge on a capacitor is can be expressed as follows

$$
\mathrm{Q}=\mathrm{C} * \mathrm{~V} \text {, }
$$

where, $\mathrm{C}=$ capacity, $\mathrm{V}=$ voltage

The time constant for $\mathrm{RC}$ is defined as

$$
\mathrm{T}=\mathrm{R} * \mathrm{C}
$$

where, $\mathrm{C}=$ capacity, $\mathrm{R}=$ series resistance.

Since a capacitor approximately takes five times of time constant to be fully charged, it is important to keep the time constant minimum. However, according to (4), to store sufficient energy for the receiver's operation, it is important to keep adequate capacity and terminal voltage in the setup. Since the terminal voltage of the capacitor is equivalent to the PV cell terminal voltage, which is static under the proper illumination, the capacitor's capacity should be selected to satisfy the energy requirement. Hence, consideration of the trade-off between charging time and the charge capacity was essential to making the selection of capacitor values

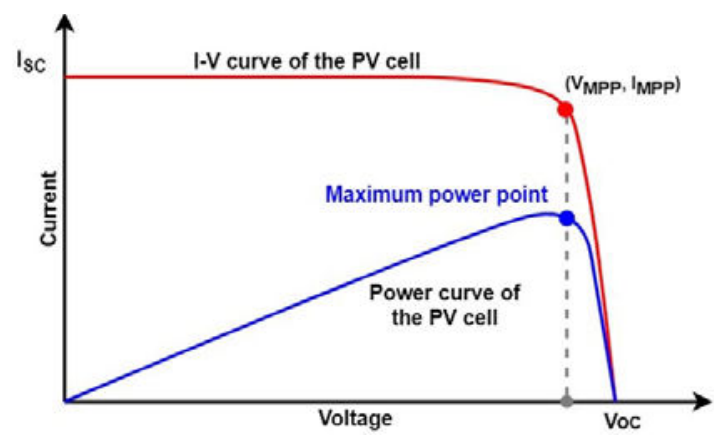

Fig.2. I-V curve of a PV cell. 


\section{DESIGN OF A LIOT PROTOTYPE}

For the LIoT concept demonstration, a LIoT-based product information label was designed and developed as a prototype. The basic structure of this smart label design is depicted in Fig.3.

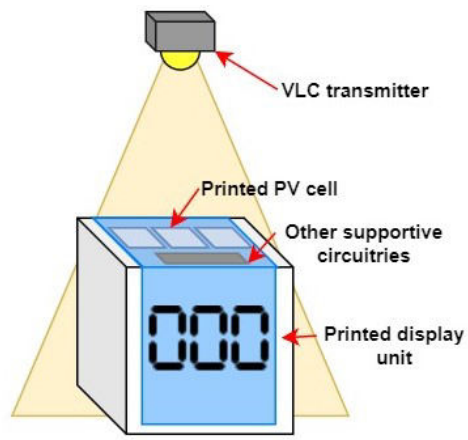

Fig.3. LIoT based product information label design.

The prototype node was designed to operate in a broadcast communication mode, and it was equipped with a display unit to display the output of the received data. The system is designed to have the capability of addressing multiple LIoT nodes scenario using the same transmitter (multi-user operation).

\section{A. Transmitter}

The structure of the prototype transmitter is illustrated in Fig.4. In this design, the transmitter unit was designed to collect input information from users and then encode and modulate the data.

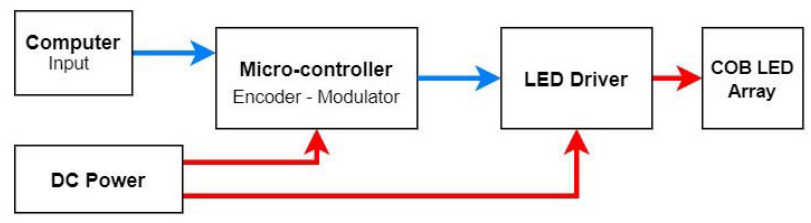

Fig.4. Design of the transmitter unit.

After that, a voltage waveform will be generated by MCU according to the modulation scheme, and then it will be fed into the LED driver. The LED driver controls the light sources according to the voltage waveform.

For implementation, Pulse-position modulation (PPM) was used at the transmitter. In order to minimize the ambient noise from the VLC channel, the pulse symbols were generated using a $38 \mathrm{kHz}$ carrier signal. The bit representation of the selected modulation scheme is depicted in Fig.5.

The modulated signal consists of 48 bits, which contains address bits and payload data bits for supporting multiuser communication for multiple nodes. The final waveform output from the MCU is shown in Fig. 6. For the implementation, an ATmega328P based Arduino Uno development board was used
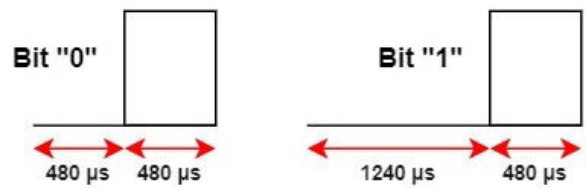

Fig.5. Bit representation of PPM modulation.

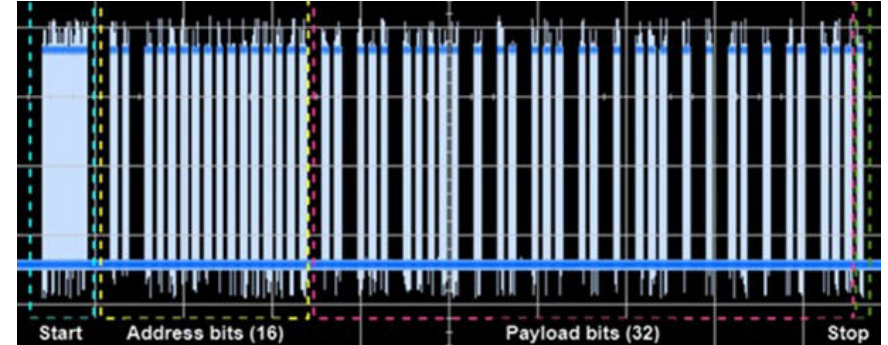

Fig.6. Output waveform from the transmitter.

as the MCU for the prototype. By considering the key parameters such as rise and fall time, drain current limitation, and TTL level signal compatibility at gate-source, IRF520 N channel MOSFET based LED driver designed was used to control the LED sources.

The LED driver unit was designed to provide sufficient modulation depth for the prototype receiver's convenient operation. The design of the LED driver is depicted in Fig.7. When selecting the LED lighting sources, the wavelengths and their efficiency on the PV panels were considered. Based on the obtained colour temperature using Wien's law and other factors such as colour suitability for indoor illumination, 5700K COB LED sources were selected.

The transmitting algorithm was designed to provide sufficient illumination to PV cells and to the indoor environment by introducing two transmitting modes. The transmission schemes were named as data signal mode and idle mode. In data signal mode, the illumination of the transmitting LEDs varies according to the modulated signal voltage. On the other hand, the system is switched to idle mode when there is not any transmitting operation going on. The voltage level variation at MCU output for two different schemes is shown in Fig.8. In this way, the receiver was expected to minimize the voltage fluctuation during the transmitting mode and provide maximum illumination performance of the LEDs on the PV cell while it is on the idle mode.

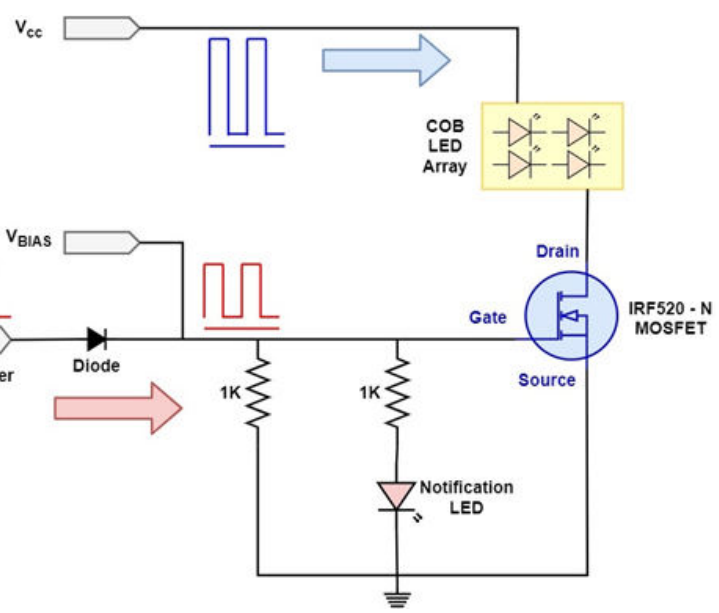

Fig.7. Design of the LED driver circuit arrangement. 


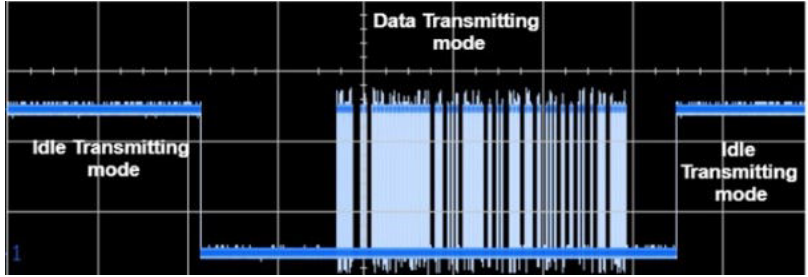

Fig.8. Voltage level variation for different transmitting schemes.

\section{B. Receiver}

The receiver circuit was designed to receive and filter out the $38 \mathrm{kHz}$ modulated data signal from the VLC channel. As depicted in Fig.9, a photodiode was used to detect the optical signal, and trans-impedance and automatic gain control amplifiers were used to convert and enhance the received signal. A bandpass filter was used to filter out the data signal and minimize the noise effects. Thereafter, the enhanced signal was fed into the MCU's ADC in order to demodulate end decode the received data.

For the implementation, a PIN photodiode was used as the optical detector. VSOP38338 IC was used as the amplifying and filtering unit. For low power consumption, ATmega328P based Arduino mini development board was used as the MCU unit. For the display unit of the node, low power consuming bi-stable display technology was selected. A printed electronic-based electrochromic seven segment display made by Ynvisible was used as the display of the LIoT node. The electrochromic printed seven segment display is shown in Fig. 10.

The energy harvesting unit was designed to provide sufficient energy for the node while keeping the size of the circuit to a minimum. In order to be easily applicable for any LIoT application, flexible printed PV cells were used for the design. For the prototype, the feasibility of two different types of printed PV cells was tested. Gravure printed perovskite PV cells developed by the technical research centre of Finland and printed organic PV cells developed by infinity PV were tested on the prototype design. The type of printed PV cells used are shown in Fig.11.

The rest of the structure of the energy harvesting unit was developed as in Fig.12. For the implementation of the circuit, the LTC 3588 energy harvesting IC was used.

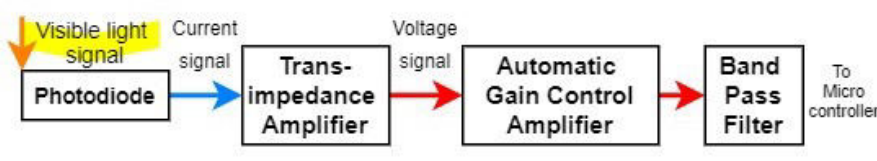

Fig.9. Receiving circuit structure.

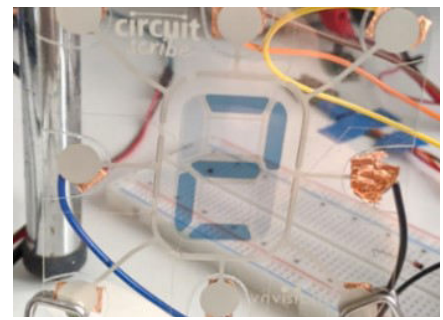

Fig.10. Electrochromic seven segment display developed by Ynvisible.

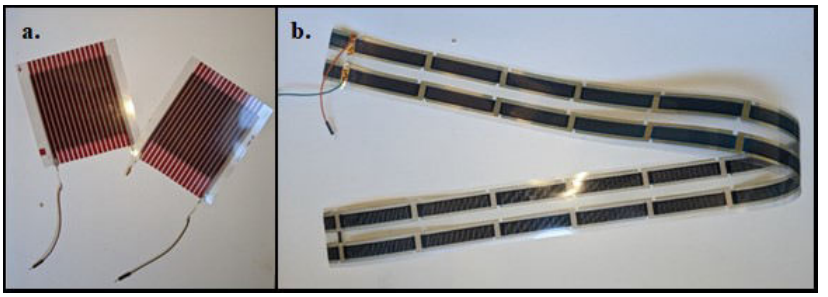

Fig. 11. a) Gravure printed perovskite PV cells developed by VTT (the technical research center of Finland) b) printed organic PV cells developed by Infinity PV.

In this design, the output from PV cells was stored in the supercapacitor arrangement. In this way, sufficient power for the node's operation will be stored in the supercapacitors due to the low current drawing nature of the energy harvesting IC. The internal capacitor was designed to provide the energy required for the connected load directly. Whenever the internal capacitor's voltage goes below the configured value, the IC is designed to recharge the internal capacitor from supercapacitor energy storage. In the implementation, to minimize the power loss during the energy storing stage, supercapacitors with low leaky currents were selected.

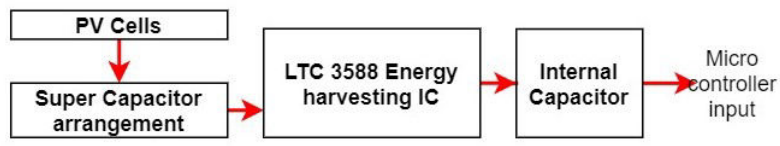

Fig.12. Energy harvesting circuit with an energy harvester IC.

\section{PERFORMANCE EVALUATION}

\section{A. BER performance}

The BER performance of the receiving circuit was measured under two different lighting scenarios. Initially, the setup was arranged under sunlight illumination (300 lux) condition and then indoor light (50 lux) condition. The receiving circuit was placed directly under the transmitter lights and then the distance between the transmitter and receiver varied. The obtained measurement is illustrated in Fig. 13

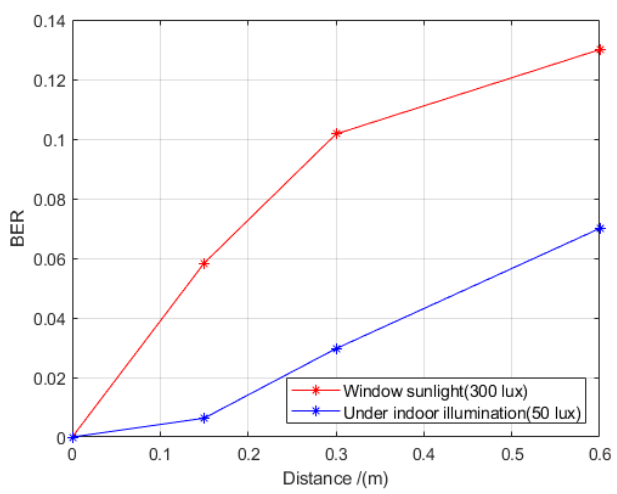

Fig.13. BER vs distance under different environment.

According to results, it can be observed that the prototype performs better in terms of BER when the distance between transmitter and receiver is below $0.2 \mathrm{~m}$. It is an acceptable distance range for the prototype's desired application. 


\section{B. SNR variation}

For the measurements, the following setup was arranged. Initially, the PV cells were placed near a window where indirect sunlight (300 lx) interferes. The transmitter was placed directly above the receiver, and then the transmitting distance was gradually changed to obtain the SNR variation. The obtained results for the experiment are shown in Fig.14.

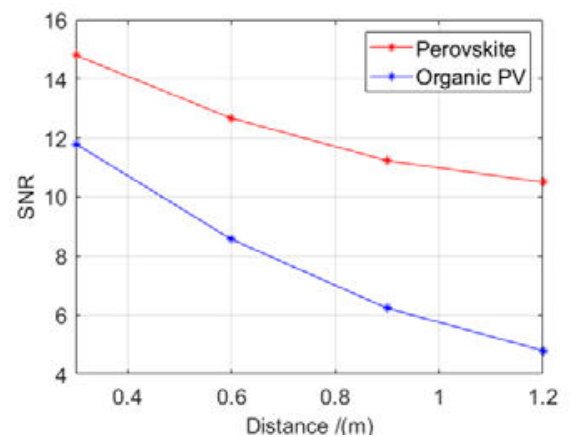

Fig.14. SNR variation with distance in an indoor sunlight environment.

Then the experiment was repeated without the effect of sunlight. This time only the transmitter illumination was directed to the receiver. The obtained results for the experiment are illustrated in Fig.15. The results from both experiments revealed that perovskite cells have better SNR performance compared to the OPV cells.

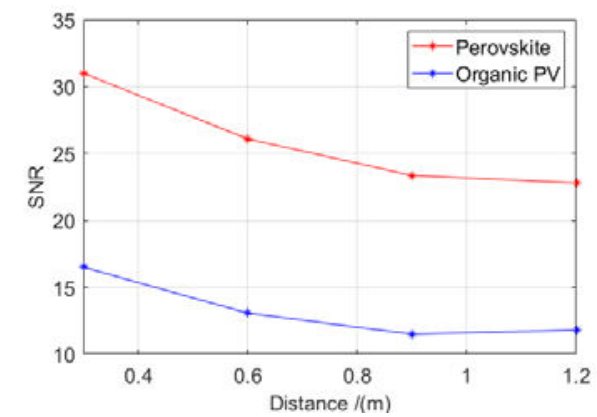

Fig.15. SNR variation with distance with only-transmitter illumination.

\section{Frequency response}

The setup for frequency response analysis of the PV cells was arranged as follows. Initially, PV cells were placed $0.2 \mathrm{~m}$ directly below the transmitter. Thereafter, a square pulse signal was transmitted, and received SNR was measured. The procedure was repeated with different frequencies for different PV cell types. The obtained results are plotted in Fig.16.

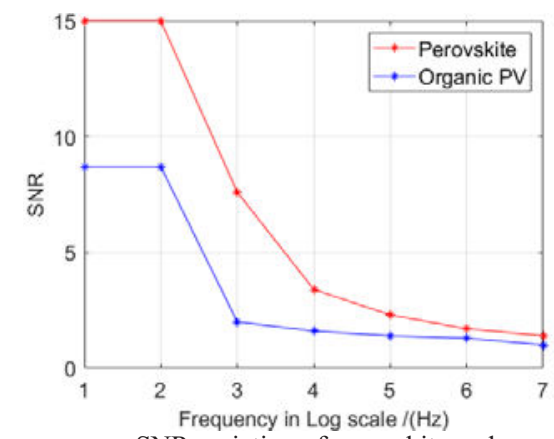

Fig.16. Frequency vs SNR variation of perovskite and organic PV cells.
The graph shows that increasing frequency results gradually decreasing SNR values on both different PE PV technologies. Moreover, it reveals that both perovskite and organic PV cell technologies have low-frequency response characteristics, and they are not suitable for high-speed data receiving purposes.

\section{Energy harvesting performance}

The energy harvesting performance of the prototype was measured under the transmitting schemes which were used in the prototype algorithm. The PV panels were positioned $0.3 \mathrm{~m}$ below the transmitter, and by varying the distance between the transmitter and PV cells, the open-circuit voltage per square of the PV cells was measured. The obtained results for the experiment are plotted in Fig. 17

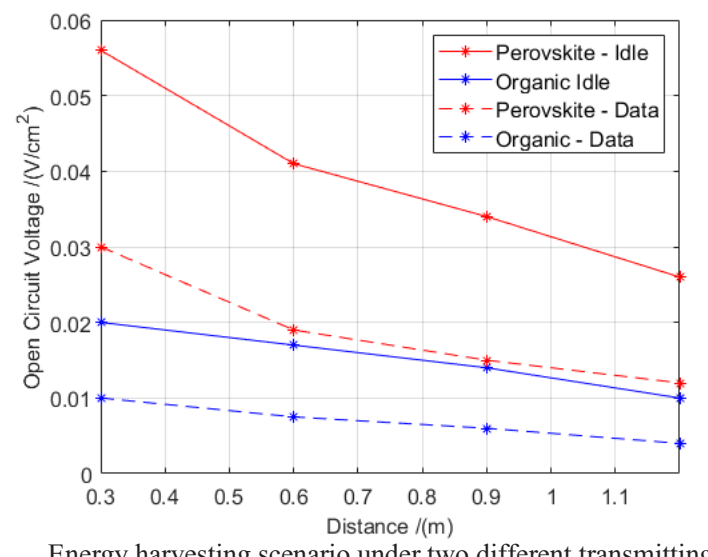

Fig.17. Energy harvesting scenario under two different transmitting schemes.

The graph results suggest that the idle transmitting scheme can produce more open circuit voltage on printed PV cells, while perovskite printed PV cells can harvest more energy compared to OPV cells.

In order to measure the energy harvesting unit's performance, current ratings through the MCU's power inputs were measured. For the comparison, current readings "with" and "without" the energy harvesting IC was considered. The obtained results are summarized in table I.

TABLE I: MAXIMUM CURRENT READINGS FOR TWO DIFFERENT METHODS

\begin{tabular}{|l|l|l|}
\hline Phase & Direct connection & Harvesting IC-based \\
\hline Booting up & $4.80 \mathrm{~mA}$ & $3.10 \mathrm{~mA}$ \\
\hline Steady operation & $4.42 \mathrm{~mA}$ & $3.00 \mathrm{~mA}$ \\
\hline
\end{tabular}

Based on the above results, it can be observed that the energy harvesting IC-based approach managed to reduce 35\% of the maximum drawing current during the bootup phase while saving $32 \%$ of the maximum drawing current during the steady operating phase. Therefore, it can be concluded that the energy harvesting IC's algorithm managed to spread the peak requirement of current along the time-axis with the help of the supercapacitor energy. 


\section{CONCLUSION}

In summary, this paper demonstrated the LIoT concept by designing and implementing a light-based energy autonomous IoT node. The applicability of the highly promising yet not fully mature PE was considered throughout the development process. For the purpose of introducing an optimum transmission strategy for LIoT nodes, the performance of two different VLC-friendly transmitting schemes was tested, compared, and based on their results; two transmission schemes were selected for the implementation. On the other hand, two commercially available OPV and perovskite PV cell technologies were tested for both energy harvesting and signal receiving, and the corresponding results revealed that the perovskite PV cell has a higher PV energy conversion efficiency, which leads to smaller PV area requirements in the design. However, the frequency response of the PV technologies showed that both PE PV technologies were unable to reach higher frequency responses, making them unusable for high data rate VLC receiving purposes. The study revealed that a general-purpose energy harvesting IC was a viable candidate for the energy harvesting process of this application due to its de-spreading effect of the peak current along the time axis. This minimized the peak current drawn from the PV panels and minimized the terminal voltage drop. In conclusion, the results of individual design segments showed that as the concept of PEbased LIoT could be effectively used for the IoT designs, which can help to produce more robust energy-autonomous flexible IoT nodes in the future.

As the future work of this, implementation can be upgraded with an uplink to support full-duplex communications. Technologies like low power infrared uplink or retroreflectorbased uplink integration can be applied to enhance the quality of the concept-based applications. The power consumption of the node can be further optimized by introducing sleep mode to the nodes while they in idle, and also a novel VLC wake-up signaling scheme can be used to wake up the idle nodes. At some point in time, when PE technology becomes mature enough, a fully PE solution of a LIoT node will be developed.

\section{ACKNOWLEDGMENT}

This work was supported by the Academy of Finland 6Genesis and Photonics Research and Innovation (PREIN, decision 320168) flagship projects.

\section{REFERENCES}

[1] H. Haas, "LiFi is a paradigm-shifting 5G technology," vol. 3, pp. 26-31, 2018.

[2] H. Haas, "Introduction to indoor networking concepts and challenges in LiFi," Journal of Optical Communications and Networking, vol. 12, no. 2, p. 190, 2019.
[3] H. Chun, "Wide-Area Coverage $35 \mathrm{~Gb} / \mathrm{s}$ Visible Light Communications Link for Indoor Wireless Applications," Scientific Reports, vol. 9, no. 1, 2019.

[4] T. Perković, "BlinkComm: Initialization of IoT Devices Using Visible Light Communication," Wireless Communications and Mobile Computing, pp. 1-16, 2018.

[5] L. Chettri, "A Comprehensive Survey on Internet of Things (IoT) Toward 5G Wireless Systems," IEEE Internet of Things Journal, vol. 7, no. 1, pp. 16-32, 2020..

[6] Rolf Arne Kjellby, Linga Reddy Cenkeramaddi, Thor Eirik, Soumya J, "Self-powered IoT Device based on Energy," in IEEE International Conference on Advanced Networks and Telecommunications Systems, 2018

[7] R. Vullers, "Micropower energy harvesting," Solid-State Electronics, vol. 53, no. 7, pp. 684-693, 2009.

[8] Afghan Syeda Adila, Almusawi Husam, Géza Husi, "Towards the SelfPowered Internet of Things (IoT) by Energy Harvesting: Trends and Technologies for Green IoT," in 2nd International Symposium on Smallscale Intelligent Manufacturing Systems (SIMS), 2018.

[9] Marcos Katz, Dominic O'Brien, "Exploiting novel concepts for visible light communications: from light-based IoT to living surfaces," Optik International Journal for Light and Electron Optics, October 2019.

[10] S. \&. H. \&. M. M. \&. M. \&. S. R. Pervez,, "LiFi: The Future for Indoor Wireless Data Communication," International Journal of Scientific and Engineering Research,, vol. 7, pp. 71-79, 2016.

[11] Tsung-Ching Huang*, Kwang-Ting Chengy, Raymond Beausoleil, Printed Circuits on Flexible Substrates:, Hewlett Packard Labs, Palo Alto, USA, 2016.

[12] Z. Cui,, "Printing practice for the fabrication of flexible and stretchable electronics," Science China Technological Sciences, vol. 62, no. 2, pp. 224-232, 2019.

[13] F. C. J. Torrisi,, "Electrifying inks with 2D materials," Nature Nanotech, pp. 738-739, 2014.

[14] Young Yun Kim, Tae-Youl Yang, Riikka Suhonen, Marja Välimäki, Tiina Maaninen, "Gravure-Printed Flexible Perovskite Solar Cells," Toward advancedscience, 2019.

[15] Y. Li, N.J. Grabham, S.P. Beeby, M.J. Tudor, "The effect of the type of illumination on the energy harvesting performance of solar cells," Elsevier, January 2015.

[16] L. A. A. Kring, "Spectroscopy," university of Georgia, Athens, Georgia 30602..

[17] Trio Adiono, Syifaul Fuada, "Optical Interference Noise Filtering over Visible Light Communication System," in 2017 International Symposium on Nonlinear Theory and Its Applications, 2017, Cancun, Mexico.

[18] Y. Zhao, "Design of Visible Light Communication Receiver for On-Off Keying Modulation by Adaptive Minimum-Voltage Cancelation," Engineering Journal, vol. 17, no. 4, pp. 125-130, 2013.

[19] Fu-Liang Chang, Wei-Wen Hu, Da-Huei Lee, Chao-Tang Yu, "Design and implementation of anti low-frequency noise in visible light communications," in Proceedings of the 2017 IEEE International Conference on Applied System Innovation, 2017.

[20] Teng-ChunWuYean-SanLongShu-TsungHsuEn-YunWang,, "Efficiency Rating of Various PV Technologies under Different Indoor Lighting Conditions,", in proceedings of the SNEC 11th International Photovoltaic Power Generation Conference \& Exhibition,SNEC 2017 Scientific Conference,, September 2017,

[21] "“electronics-tutorials," [Online]. Available: https://www.electronicstutorials.ws/rc/rc_1.html.," [Online 